\title{
Reduced ADAMTS13 activity is associated with an ADAMTS13 SNP, fever and microparticles in a malaria-like model
}

\author{
Sirima Kraisin ${ }^{1}$, Attakorn Palasuwan ${ }^{1}$, Supaluk Popruk ${ }^{2}$ and Duangdao Nantakomol ${ }^{1 *}$
}

\begin{abstract}
Background: Severe falciparum malaria (SM) remains a major cause of death in tropical countries. The reduced activity of ADAMTS13, increasing levels of ultra-large von Willebrand factor (ULWWF) in SM patients, are assumed as factors that intensify disease severity. However, the reason why ADAMTS13 activity is reduced in SM remains unclear.
\end{abstract}

Objectives: To investigate whether rs4962153, febrile temperature, and microparticles, contribute to reduced ADAMTS13 activity.

Methods: Genotypic association of rs4962153 with ADAMTS13 antigen and activity was examined in 362 healthy Thai participants. The collagen binding assay was used to study the effects of febrile temperature and microparticles on ADAMTS13 activity.

Results: ADAMTS13 antigen and activity were decreased in participants with AA genotype, compared to AG and GG (antigen: $p$-value $=0.014$, and $<0.001$; activity: $p$-value $=0.036$, and $<0.002$, respectively). There was significantly reduced ADAMTS13 antigen in AG compared to GG ( $p$-value $=0.013$ ), but not in ADAMTS13 activity ( $p$-value $=0.082$ ). The number of rs4962153 A alleles correlated with the reduced level of antigen and activity ( $p$-value $<0.001$ and $p$-value $=0.001$, respectively). MPs showed an inhibitory effect on ADAMTS13 activity ( $p$-value $=0.025$ ). Finally,

ADAMTS13 activity was decreased in a temperature and time-dependent manner. The interaction between these two factors was also observed ( $p$-value $<0.001$ ).

Conclusions: These findings suggest that the A allele of rs4962153, MPs, and febrile temperature, contribute to reduce ADAMTS13 activity in plasma. These data are useful in malaria or other diseases with reduced ADAMTS13 activity.

Keywords: ADAMTS13, Cell-derived microparticles, Fever, Plasmodium falciparum, Malaria, Single nucleotide polymorphism

\section{Background}

Plasmodium falciparum malaria is a life-threatening disease in tropical countries, which causes approximately 500 million clinical cases, resulting in about 650,000 deaths each year [1]. Cerebral malaria $(\mathrm{CM})$ is the most serious complication of severe malaria (SM) contributing to cause of death. Central pathophysiology of CM is the sequestration of parasitized red blood cells (PRBCs) in the cerebral microvasculature, which leads to microvascular obstruction and endothelial activation, including the release of von

\footnotetext{
* Correspondence: nantadao@gmail.com

'Department of Clinical Microscopy, Faculty of Allied Health Sciences,

Chulalongkorn University, Bangkok, Thailand

Full list of author information is available at the end of the article
}

Willebrand factor (vWF) from its storage site in the endothelium, Weibel-Palade bodies [2]. However, the pathogenesis of CM remains poorly understood.

vWF is a large multimeric plasma glycoprotein that plays an important role in primary haemostasis by mediating the adhesion of platelets to sites of vascular injury. Normally, vWF and platelet-decorated vWF string is cleaved and regulated by an endogenous protease, a disintegrin and metalloproteinase with a thrombospondin type 1 motif, member 13 (ADAMTS13). Interestingly, the level of vWF and its propeptide are increased in SM patients compared to uncomplicated malaria and healthy control, and the increased level related with disease severity [3]. Moreover, there was abnormal ULVWF, a highly active 
form of vWF contributing to large platelet adhesion and aggregation to the vessel wall, in SM and CM patients rather than normal control $[4,5]$. These data correspond with the reduced activity of ADAMTS13 in SM and CM patients $[4,6]$. Furthermore, most symptoms of CM including fever, renal failure, microangiopathic haemolytic anaemia, neurological deficits and thrombocytopaenia, are commonly found in thrombotic thrombocytopaenic purpura (TTP), a rare life-threatening disease, which is caused by a congenital or acquired deficiency of ADAMTS13. Consequently, the factors causing the reduction of ADAMTS13 antigen and activity are, therefore, important in the pathogenesis of SM and CM.

It is well known that mutations in ADAMTS13 gene contribute congenital TTP. A relatively large number of mutations and polymorphisms have been identified in ADAMTS13 [7]. Recent study reported that the A allele of an intronic SNP, rs4962153, is associated with decreased risk of CM [8]. However, the effect of this SNP to expression level and activity of ADAMTS13 have not been studied.

A number of studies reported that level of MPs is increased in plasma with SM and CM, and correlated with disease severity [9-11]. MPs are vesicular particles released from blood cells, from which their functionalities are dependent on their original cell types. Surprisingly,MPs enhance the function of VWF during vascular injury, causing formation and more stability of platelet aggregation [12]. Although, some studies suggested that MPs derived from endothelial cells and platelets seem to have inhibitory capacity on ADAMTS13 activity [13,14], those are very preliminary studies. Activity of ADAMTS13 in plasma is quite stable. No significant decrease in ADAMTS13 activity is observed during storage at room temperature for up to $48 \mathrm{hr}$ and $37^{\circ} \mathrm{C}$ for longer than one week; however, whether febrile temperature has any effect on ADAMTS13 activity has not been reported $[15,16]$. As fever is one of the symptoms established in both of TTP and SM, suggesting that effects of febrile temperature to ADAMTS13 activity should be investigated.

The aim of the present study was, therefore, to examine the effects from interesting factors that were found in SM on ADAMTS13. Genotypic association between rs4962153 and ADAMTS13 was explored in the Thai population. In addition, ADAMTS13 activity was measured in MPsenriched plasma compared to MPs-depleted plasma. Finally, the activity of ADAMTS13 was determined in febrile-like plasma samples compared to normal plasma.

\section{Methods}

\section{Subjects}

A total of 362 healthy Thai people aged 18-44 years old were recruited in this study (254 females and 108 males). Those participants were healthy without fever, inflammation, infection, and taking of any antiinflammatory medicines for at least seven days before blood collection. Furthermore, the participants had no medical history of any coagulopathies including haemophilia, thromboembolism, bleeding disorders, heart diseases, and severe inflammatory diseases. This study has approved by the Ethics Review Committee for Research Involving Human Research Subjects, Health Science Group, Chulalongkorn University, Thailand. Written informed consent was obtained from all participants.

\section{DNA extraction}

Genomic DNA was extracted from EDTA blood using a FavorPrep $^{\mathrm{Tm}}$ FABGK genomic DNA Extraction mini kit according to the manufacturer's instruction (Favorgen, Australia).

\section{Genotyping}

rs4962153 (g.41635A > G), a significant SNP of ADAMTS13 gene associated with cerebral malaria [8], was genotyped by TaqMan SNP Genotyping Assay for 362 healthy participants using StepOnePlus ${ }^{\text {TM }}$ Real-Time PCR systems (Applied Biosystems, USA).

\section{Genotypic association study}

34 of 362 participants were selected by convenience sampling and classified by their genotypes into three groups, which are GG, AG, or AA $(19,13$ and two samples, respectively). Citrated plasma samples were collected from those participants and then the level of ADAMTS13 antigen and activity were performed using commercial kits (Quantikine Human ADAMTS13 ELISA kit; R\&D Systems Inc., U.S.A, and Technozyme ADAMTS13 activity ELISA; Technoclone, Austria). The level of ADAMTS13 antigen and activity in each sample was determined in duplicate.

\section{Effect of microparticles on ADAMTS13 activity}

Nineteen participants with genotype GG of rs496215. Three who presented the range of ADAMTS13 activity from 80 to $90 \%$ were enrolled in this study. The plasma samples were first prepared from platelet rich plasma (PRP), which was carefully collected after centrifugation at $250 \mathrm{~g}$ for $15 \mathrm{~min}$ at room temperature. The PRP was then centrifuged at $13,000 \mathrm{~g}$ for $3 \mathrm{~min}$ at room temperature. The MPs-enriched plasma was taken, which is referred to as 'Non-filtered (NF)' plasma throughout this article. The residual plasma was exhaustively filtered using Mimisart ${ }^{\circledR}$ Syringe filter hydrophilic with a pore size of $0.2 \mu \mathrm{m}$ (Sartorius Stedim Biotech, Germany) to deplete MPs. The MPs-depleted plasma will be indicated throughout this article as 'Filtered (F)' plasma. Flow cytometry was used to confirm the events of MPs in both of NF and F plasma samples as described previously [11]. The ADAMTS13 
activity was measured by the Collagen Binding Assay (CBA) as described below.

\section{Effect of febrile temperature on ADAMTS13 activity}

The same citrated plasma samples from 30 participants as recruited in MPs test were used in this study. The aliquot samples were separately incubated at three different temperatures which are $37^{\circ} \mathrm{C}, 38^{\circ} \mathrm{C}$ and $39^{\circ} \mathrm{C}$ for $0,3,6,12,24$ and $48 \mathrm{hr}$ in each temperature. The ADAMTS13 activity was measured by the CBA.

\section{Measurement of ADAMTS13 activity by the collagen binding assay}

To measure the effects of febrile temperature and MPs, the ADAMTS13 activity was analyzed in 30 citrated plasma samples by CBA, as described with slight modifications [17]. Importantly, febrile plasma samples which were previously incubated at $38^{\circ} \mathrm{C}$ and $39^{\circ} \mathrm{C}$ in plasma preparation process would be incubated at $38^{\circ} \mathrm{C}$ and $39^{\circ} \mathrm{C}$ instead of $37^{\circ} \mathrm{C}$ in digestion step, respectively. The chromogenic reaction in the final process was performed by addition of $100 \mu \mathrm{l}$ of substrate o-phenylenediamine dihydrochloride (5 mg) (Med OPD P6912, Sigma Aldrich, USA). The ELISA reaction was stopped by addition of $100 \mu \mathrm{H}_{2} \mathrm{SO}_{4}$ (3\%) and absorbance was read at $492 \mathrm{~nm}$ with reference length at $620 \mathrm{~nm}$ using Sunrise ${ }^{\text {Tx }}$ absorbance reader (Tecan Group Ltd. Switzerland). The ADAMTS13 activity in each sample was determined in duplicate.

Normal pooled plasma (NPP), which was collected from 30 healthy participants was used to generate a calibration curve. The NPP was diluted in to 7 dilutions, $1: 5,1: 10,1: 20,1: 40,1: 80,1: 160$ and $1: 320$ in $1.5 \mathrm{M}$ urea, $5 \mathrm{mM}$ Tris, $\mathrm{pH}$ 8.0. The ADAMTS13 activity was read from the sigmoid-like calibration curve using point-topoint plot. The NPP dilution of 1:10 was defined as $107 \%$ of ADAMTS13 activity.

\section{Statistical analyses}

Data are presented as means \pm SEMs and analyzed by the software SPSS version 17.0 (SPSS Inc.). Statistical significances in genotypic association and effect of febrile condition were assessed by One-way ANOVA and Two-way ANOVA with repeated measures, respectively. A correction for multiple comparisons between individual groups was made using Tukey-HSD Post-Hoc analysis. Effect of MPs on ADAMTS13 was assessed using the paired $t$-test. Linear regression was used to describe the correlation between number of A allele of rs4962153 and ADAMTS13 antigen as well as activity as previously described [18]. Briefly, genotypes of all participants were coded as $\mathrm{GG}=0, \mathrm{AG}=1$, and $\mathrm{AA}=2$, according to the number of $\mathrm{A}$ allele. The $p$-value of less than 0.05 was considered to be of statistical significance.

\section{Results}

Frequency of rs4962153 SNP in Thai population

Among the 362 healthy Thai people, the frequencies of rs4962153 of ADAMTS13 gene were 337 (93.10\%) of genotype GG, 23 (6.35\%) of genotype AG, and 2 (0.55\%) of genotype AA. The minor allele frequency of this SNP was $3.98 \%$, whereas heterozygote frequency was $6.35 \%$.

\section{Genotypic association study}

ADAMTS13 antigens were remarkably decreased in participants with the AA genotypes of rs4962153 (mean \pm SEM = $327.26 \pm 62.53 \mathrm{ng} / \mathrm{ml}$ ), compared to AG and GG genotypes (mean $\pm \mathrm{SEM}=568.02 \pm 32.54 \mathrm{ng} / \mathrm{ml}, p$-value $=0.014$, and mean $\pm \mathrm{SEM}=683.91 \pm 22.43 \mathrm{ng} / \mathrm{ml}, \quad p$-value $<0.001$, respectively). Similarly, the participants with AA genotype represent the significant reduction of ADAMTS13 activity (mean $\pm \mathrm{SEM}=58.26 \pm 5.83 \%$ ), compared to the individuals with $A G$ (mean $\pm S E M=84.78 \pm 3.5 \%, p$-value $=$ 0.036 ), and GG genotypes (mean $\pm \mathrm{SEM}=95.53 \pm 3.25 \%$, $p$-value $=0.002$ ). It should be noted that the comparison of ADAMTS13 antigen between individuals with GG and AG was significantly different, whilst no significant difference of ADAMTS13 activity was observed between those individuals ( $p$-value $=0.013$ and 0.082 , respectively; Figure 1 ).

\section{Effect of microparticles on ADAMTS13 activity}

The median (range) of MPs was significantly higher in NF plasma $(2,546(1198-3,432 / \mu \mathrm{l}))$ than F plasma (5 (2$10 / \mu \mathrm{l}), p$-value $<0.01)$. Interestingly, ADAMTS13 activity in NF plasma was significantly decreased (mean $\pm \mathrm{SEM}=$ $86.06 \pm 6.94 \%$ ), when compared to $\mathrm{F}$ plasma (mean \pm $\mathrm{SEM}=104.03 \pm 10.78 \%, p$-value $=0.025$; Figure 2 ). These data could support the inhibitory role of MPs on ADAMTS13 activity in plasma.

\section{Effect of febrile temperature on ADAMTS13 activity}

To investigate the effects from febrile temperatures, incubation times, including the interaction effect between temperatures and incubation time on ADAMTS13 activity, we used ANOVA with repeated measures (Table 1). ADAMTS13 activities were different for the 6 incubations times $(p$-value $<0.001)$. The significant differences in ADAMTS13 activity observed in plasma samples, incubated at $37^{\circ} \mathrm{C}, 38^{\circ} \mathrm{C}$ and $39^{\circ} \mathrm{C}$ ( $p$-value $<0.001$ ), indicate that temperature influences ADAMTS13 activity. Moreover, incubation time and temperature also showed the significant interaction effect on ADAMTS13 activity ( $p$-value $<0.001)$. This effect means that the level of ADAMTS13 activities at different temperatures was different after incubation for $0 \mathrm{hr}, 3 \mathrm{hr}, 6 \mathrm{hr}, 12 \mathrm{hr}, 24 \mathrm{hr}$, and $48 \mathrm{hr}$. Indeed, following incubation at $37^{\circ} \mathrm{C}, 38^{\circ} \mathrm{C}$ and $39^{\circ} \mathrm{C}$, clear reductions in ADAMTS13 activity were observed in a time-dependent manner (Figure 3). Following $6 \mathrm{hr}$ of incubation at $37^{\circ} \mathrm{C}$, there was no significant reduction in 


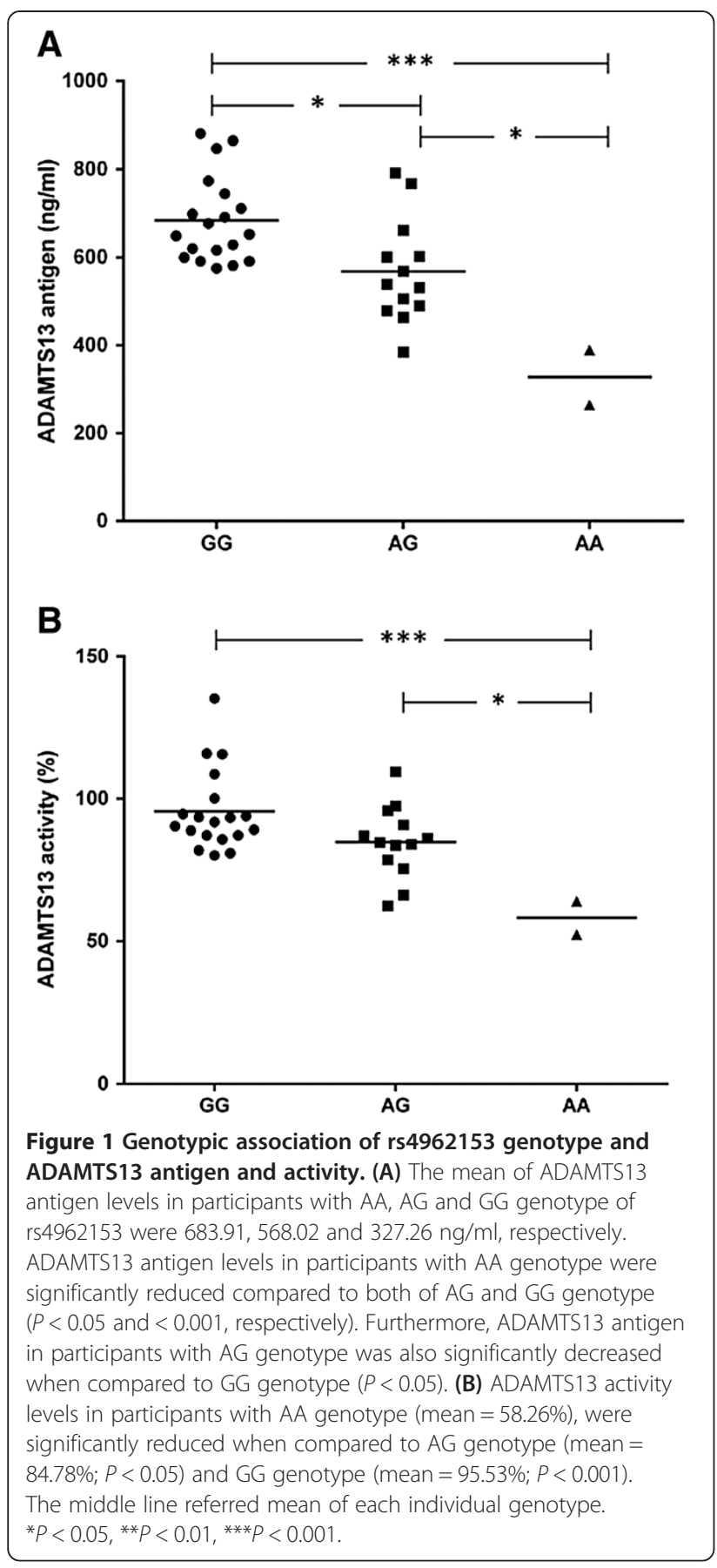

ADAMTS13 activity found when compared to 0 hr. However, after continuous incubation, the level of ADAMTS13 activity was significantly decreased at $12 \mathrm{hr}, 24 \mathrm{hr}$, and $48 \mathrm{hr}$ (Figure 3A). It should be noted that ADAMTS13 activity in normal plasma was reduced by approximately $50 \%$ after incubation at $37^{\circ} \mathrm{C}$ for $48 \mathrm{hr}$. Similarly, the ADAMTS13 activity was initially declined after incubation at $38^{\circ} \mathrm{C}$ for $3 \mathrm{hr}$, compared to $0 \mathrm{hr}$. More significant reduction corresponded with increased incubation time; the level of ADAMTS13 activity following incubation for

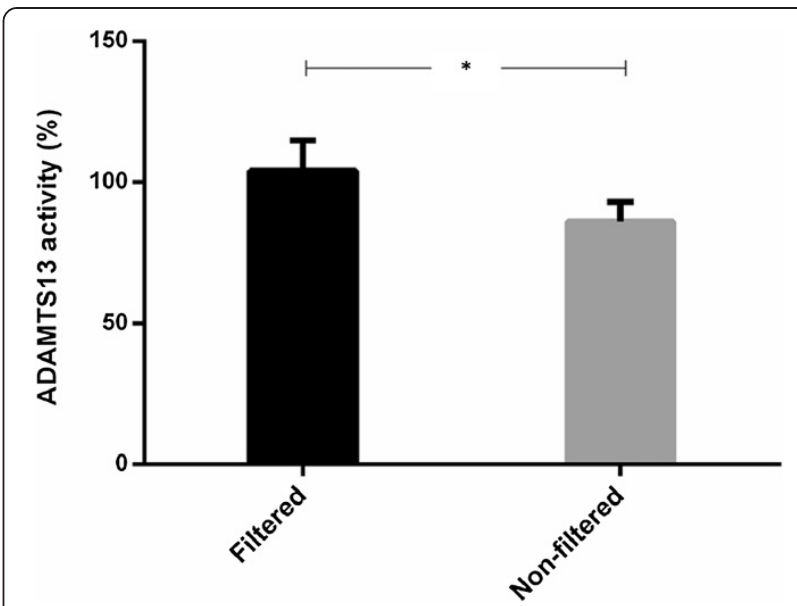

Figure 2 Inhibitory effect of MPs on ADAMTS13 activity. Non-filtered plasma samples presented ADAMTS13 activity $86.06 \%$, which was significant lower than the filtered-plasma samples (mean $=104.03 \%)$. ${ }^{*} P<0.05$.

$48 \mathrm{hr}$ at $38^{\circ} \mathrm{C}$ was decreased compared to $0 \mathrm{hr}$, by approximately $30 \%$. These data might indicate that ADAMTS13 activity is more stable in $38^{\circ} \mathrm{C}$ than $37^{\circ} \mathrm{C}$ after incubation for $48 \mathrm{hr}$ (Figure 3B). Moreover, the incubation at $39^{\circ} \mathrm{C}$ represented the strongest reduction of ADAMTS13 activity, as the inhibitory effect was initially observed in plasma samples that were not incubated in preparation process $(0 \mathrm{hr})$. The inhibitory effect remains after incubation for $3 \mathrm{hr}$ to $48 \mathrm{hr}$ in a time-dependent manner. Interestingly, ADAMTS13 was almost absent after the incubation for $48 \mathrm{hr}$ under $39^{\circ} \mathrm{C}$ (mean $\pm \mathrm{SEM}=2.01 \pm 1.01 \%$; Figure $3 \mathrm{C}$ ). These data led us to assume that the level of ADAMTS13 activity in plasma samples without incubation in the preparation process $(0 \mathrm{hr})$ was declined by approximately $30 \%$ and $60 \%$ after incubated at $38^{\circ} \mathrm{C}$ and $39^{\circ} \mathrm{C}$ during the digesting process in $\mathrm{CBA}$, respectively, compared to the same plasma incubated under normal conditions $\left(37^{\circ} \mathrm{C}\right)$.

Clear evidence of the temperature-dependent inhibitory effect was observed (Figure 3D). ADAMTS13 activities were most inhibited at $39^{\circ} \mathrm{C}$, and the least inhibitory effect observed at $37^{\circ} \mathrm{C}$, with moderate inhibition at $38^{\circ} \mathrm{C}$. To confirm these events, Post-Hoc analysis was used to assess the differences of ADAMTS13 activity at all temperatures, $37^{\circ} \mathrm{C}$ versus $38^{\circ} \mathrm{C}, 37^{\circ} \mathrm{C}$ versus $39^{\circ} \mathrm{C}$, and $38^{\circ} \mathrm{C}$ versus $39^{\circ} \mathrm{C}$, in each incubation time. The result showed that there were

Table 1 Effects of Febrile temperatures and Incubation time to ADAMTS13 activity*

\begin{tabular}{lccc}
\hline Factors & df & F & p-value \\
\hline Incubation time & 5 & 40.086 & $<0.001$ \\
Temperature & 2 & 138.35 & $<0.001$ \\
Incubation time * Temperature & 10 & 8.063 & $<0.001$
\end{tabular}

*Incubation time *temperature $=$ interaction effect of temperature and incubation time; $\mathrm{df}=$ degree of freedom; $\mathrm{F}=\mathrm{F}$-test score. 


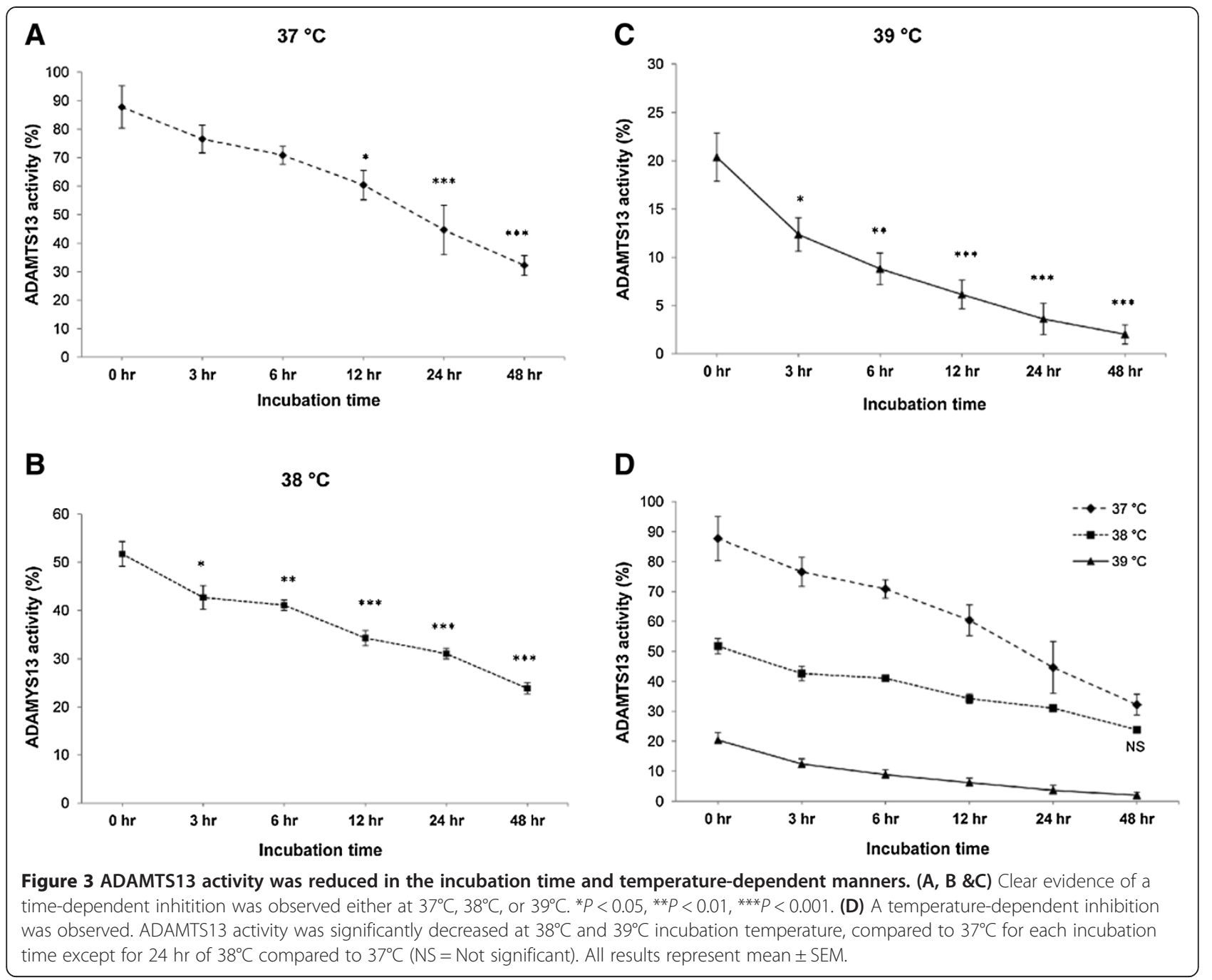

significant differences observed between ADAMTS13 activities in most comparisons at each incubation time except the comparison between $37^{\circ} \mathrm{C}$ and $38^{\circ} \mathrm{C}$ at $24 \mathrm{hr}(p$-value $=$ 0.187; Table 2).

\section{Discussion}

A number of previous studies have shown that the level of ULVWF and vWF multimers are higher in patients with
SM and CM, together with decreased antigen and activity of ADAMTS13, compared to healthy controls $[4,6]$. The present finding revealed that AA genotype of rs4962153 associated with notably reduced activity and antigen of ADAMTS13 in healthy participants, when compared to AG and GG genotypes. The number of A allele showed strong association with reduced ADAMTS13 antigen and activity, which could confirm allele A is significant.

Table 2 Level of ADAMTS13 activity in plasma incubated in normal and febrile temperature

\begin{tabular}{|c|c|c|c|c|c|c|}
\hline \multirow{2}{*}{$\begin{array}{l}\text { Incubation } \\
\text { time (hr.) }\end{array}$} & \multicolumn{3}{|c|}{$\%$ ADAMTS13 activity (mean \pm SEM*) } & \multicolumn{3}{|c|}{$p$-value (Post-Hoc Tukey HSD) } \\
\hline & $37^{\circ} \mathrm{C}$ & $38^{\circ} \mathrm{C}$ & $39^{\circ} \mathrm{C}$ & $37^{\circ} \mathrm{C}$ vs $38^{\circ} \mathrm{C}$ & $37^{\circ} \mathrm{C}$ vs $39^{\circ} \mathrm{C}$ & $38^{\circ} \mathrm{C}$ vs $39^{\circ} \mathrm{C}$ \\
\hline 0 & $87.75 \pm 7.39$ & $51.73 \pm 2.57$ & $20.36 \pm 2.49$ & $<0.001$ & $<0.001$ & 0.001 \\
\hline 3 & $76.57 \pm 4.89$ & $42.67 \pm 2.45$ & $12.36 \pm 1.73$ & $<0.001$ & $<0.001$ & $<0.001$ \\
\hline 6 & $70.87 \pm 3.11$ & $41.08 \pm 1.09$ & $8.80 \pm 1.64$ & $<0.001$ & $<0.001$ & $<0.001$ \\
\hline 12 & $60.38 \pm 5.19$ & $34.24 \pm 1.60$ & $6.14 \pm 1.49$ & $<0.001$ & $<0.001$ & $<0.001$ \\
\hline 24 & $44.65 \pm 8.65$ & $31.04 \pm 1.03$ & $3.60 \pm 1.62$ & 0.187 & $<0.001$ & 0.007 \\
\hline 48 & $32.22 \pm 3.49$ & $23.83 \pm 1.17$ & $2.01 \pm 1.01$ & 0.048 & $<0.001$ & $<0.001$ \\
\hline
\end{tabular}

*SEM = Standard Error of the Mean. 
Moreover, MPs have inhibitory effects on ADAMTS13 activity in normal plasma. Finally, febrile temperature and incubation time themselves and interaction together significantly decreased ADAMTS13 activity in temperature and time-dependent manner.

In recent years genetic variation in ADAMTS13 gene associated with congenital TTP has been reported [7]. Interestingly, a previous study has shown that an intronic SNP, rs4962153-A, was significantly associated with protection against CM in Thai patients [8]. This study was designed to investigate the genotype and allele frequency in 362 healthy Thai participants. Considering genotype frequency, AA genotype was rarely found in those participants. Correspond with minor allele frequency (A allele), which was less than $5 \%$ in this study. HapMap project revealed that this SNP is unusually found in Asian population, including Chinese, and Japanese $(5.8 \%$, and 9.9\%, respectively), whereas, commonly found in European and subSaharan African (15.6\% and 38.5\%, respectively) [19]. In this recent study, it was found that AA genotype associated with reduced ADAMTS13 antigen and activity compared to AG and GG genotypes (Figure 1). Moreover, the significant difference of ADAMTS13 antigen was also noticed in a comparison between AG and GG genotype, but not in ADAMTS13 activity. Although only two participants with AA genotype were enrolled in this study, the individuals with GG genotypes had about two times higher in both ADAMTS13 antigen and activity (2.09 and 1.64, respectively). Furthermore, the level of antigen in participants with AA genotype $(327.26 \pm 62.53 \mathrm{ng} / \mathrm{ml})$ was slightly lower than the minimal level of normal range for ADAMTS13 in citrate plasma (370-1403 ng/ml; Quantikine ${ }^{\odot}$ ELISA human ADAMTS13 manual), the activity in those participants $(58.26 \pm 5.83 \%)$ was included in reference range (40-130\%; TECHNOZYM ${ }^{\circledR}$ ADAMTS-13 Activity manual). These data may explain that even if the antigen level is low, there are some compensatory mechanisms that can support normal ADAMTS13 activity. However, a study in a larger Thai population or in other populations should be examined.

The A allele of rs4962153 was assumed to be a risk allele as it associated with the decreased ADAMTS13 antigen and activity in this present study. This finding supports a Swedish study, which reported that rs4962153-A was associated with susceptibility to ischemic stroke (IS) [20]. Therefore, rs4962153-A may reduce the ADAMTS13 activity in IS patients. On the other hand, the A allele was associated with decreased risk of CM in the previous study [8]. As the transcription level of ADAMTS13 involved with rs4962153-A is unlikely increased or decreased depending on the situation, the reported associations may be false positive in either study. However, this present study examined in healthy participants in vitro, functional factors found in malaria patients may cause the difference. To assess the results, the effect of this SNP on mRNA and/or ADAMTS13 antigen as well as its activity in malaria patients should be further investigated. The information about rs4962153 is not much investigated. It is an intronic SNP without known function. This SNP is possibly in strong linkage disequilibrium (LD) with a variant that regulates gene expression, or with a non-synonymous coding SNP. Moreover, alternative splice site spanning the location of rs4962153, including effect an enhancer/silencer encoded within the intron, should be considered.

Platelets and ECs are activated, and then apoptosis is increased in CM, which leads to MPs release in those cells. MPs are submicronic membranous rudiments characterized by the elevated exposure on the external membrane leaflet of anionic phospholipids, such as phosphatidylserine (PS), carrying on their surface proteins from the original cell types, which give them specific biological properties [21]. Platelets are able to modulate PRBC cytoadherence and PMPs represent the majority of circulating MPs. PMPs transfer platelet antigens such as PECAM-1 and CD36, which are major receptors supporting PRBC adhesion $[10,22]$. An initial process of thrombus formation via vWFplatelet interaction is the binding of platelet receptor GPIb $\alpha$ to the vWF A1 domain, resulting in the exposure of the binding and cleavage site for ADAMTS13 under shear stress [10]. The present study has found an inhibitory effect from MPs (mostly released from platelets) on ADAMTS13 activity. This finding agrees with a preliminary study, which implied that activated platelets and PMPs could regulate ADAMTS13 activity [14]. Altogether, the increased PMPs in circulation could possibly compete in binding ADAMTS13 by reducing and/or blocking its interaction with vWF, leading to conformational change and less multimeric cleavage. It seems unlikely PMPs alone strongly decrease ADAMTS13 activity as the activity in PMPs-enriched plasma remained in normal range; however, high level of PMPs together with other risk possibly contribute sturdily decrease ADAMTS13 activity and then increase risk of thrombosis.

The present data show that following incubation at $37^{\circ} \mathrm{C}$ for $48 \mathrm{hr}$, ADAMTS13 activity was slowly decreased, represent half-life about 24-48 hr in-vitro. The half-life is slightly shorter than the respective half-live (48-72 hr) observed in TTP patients [23]. This data is slightly in conflict with an in-vitro study which reported that ADAMTS13 was quite stable with half-life longer than 1 week [16]. Since the studies have been examined in different populations and under different conditions, further independent studies are needed to examine whether the findings can be confirmed. ADAMTS13 activity was reduced continuously during incubation with half-life spanning about $48 \mathrm{hr}$ and $3 \mathrm{hr}$ after incubation at $38^{\circ} \mathrm{C}$ and $39^{\circ} \mathrm{C}$, respectively. Moreover the protease activity was markedly inactivated when the temperature was increased (Figure 3). It seems likely 
that ADAMTS13 activity is inactivated depending on time and temperature. In falciparum malaria, core temperature of patients may rise as high as $42^{\circ} \mathrm{C}$. An in-vitro study revealed that fever accelerates and increases the cytoadherence of PRBCs to the major parasitized receptors, CD36 and ICAM-1 [24]. The possible mechanism may be related with the inactivated ADAMTS13 activitiy, which results insufficiently regulated the platelet-decorated ULVWF strings.

These findings show that ADAMTS13 activity is reduced in healthy Thai participants with AA genotype compared to AG and GG genotype, and seems related to a number of A alleles. Moreover, MPs and febrile temperature have the capacity to inhibit and/or inactivate ADAMTS13. The present findings are not implied only in malaria infection, but also in other diseases for example, TTP, heart disease, coagulopathies, and other inflammatory diseases.

\section{Competing interests}

The authors declare that they have no competing interests.

\section{Authors' contributions}

SK drafted the manuscript and performed experiments. AP performed data analysis and interpretation. SP collected the samples. DN involved in providing the conception, design of the study and revised the manuscript critically for intellectual content and approved the final version of the manuscript. All authors read and approved the final manuscript.

\section{Acknowledgements}

This study was supported by the Chulalongkorn University (RES560530154-HR). Miss Sirima Kraisin wish to acknowledge the Graduate Scholarship to Commemorate the 72nd Anniversary of His Majesty King Bhumibol Adulyadej of 2010 academic year, and the 90th Anniversary of Chulalongkorn University fund (Ratchadaphiseksomphot Endowment Fund) of 2012 academic year.

\section{Author details}

'Department of Clinical Microscopy, Faculty of Allied Health Sciences, Chulalongkorn University, Bangkok, Thailand. 'Department of Protozoology, Faculty of Tropical Medicine, Mahidol University, Bangkok, Thailand.

Received: 18 June 2013 Accepted: 29 December 2013

Published: 3 January 2014

\section{References}

1. Snow RW, Guerra CA, Noor AM, Myint HY, Hay SI: The global distribution of clinical episodes of Plasmodium falciparum malaria. Nature 2005, 434:214-217.

2. de Mast Q, Groot E, Lenting PJ, de Groot PG, McCall M, Sauerwein RW, Fijnheer $\mathrm{R}$, van der Ven A: Thrombocytopenia and release of activated von Willebrand Factor during early Plasmodium falciparum malaria. $J$ Infect Dis 2007, 196:622-628.

3. Hollestelle MJ, Donkor C, Mantey EA, Chakravorty SJ, Craig A, Akoto AO, O'Donnell J, van Mourik JA, Bunn J: von Willebrand factor propeptide in malaria: evidence of acute endothelial cell activation. Br J Haematol 2006, 133:562-569.

4. Larkin D, de Laat B, Jenkins PV, Bunn J, Craig AG, Terraube V, Preston RJ, Donkor C, Grau GE, van Mourik JA, O'Donnell JS: Severe Plasmodium falciparum malaria is associated with circulating ultra-large von Willebrand multimers and ADAMTS13 inhibition. PLoS Pathog 2009, 5:e1000349.

5. Bridges DJ, Bunn J, van Mourik JA, Grau G, Preston RJ, Molyneux M, Combes V, O'Donnell JS, de Laat B, Craig A: Rapid activation of endothelial cells enables Plasmodium falciparum adhesion to platelet-decorated von Willebrand factor strings. Blood 2010, 115:1472-1474.

6. Lowenberg EC, Charunwatthana P, Cohen S, van den Born BJ, Meijers JC, Yunus EB, Hassan MU, Hoque G, Maude RJ, Nuchsongsin F, Levi M, Dondorp
AM: Severe malaria is associated with a deficiency of von Willebrand factor cleaving protease, ADAMTS13. Thromb Haemost 2010, 103:181-187.

7. Lotta LA, Garagiola I, Palla R, Cairo A, Peyvandi F: ADAMTS13 mutations and polymorphisms in congenital thrombotic thrombocytopenic purpura. Hum Mutat 2010, 31:11-19.

8. Kraisin S, Naka I, Patarapotikul J, Nantakomol D, Nuchnoi P. Hananantachai H, Tsuchiya N, Ohashi J: Association of ADAMTS13 polymorphism with cerebral malaria. Malar J 2011, 10:366.

9. Combes V, Taylor TE, Juhan-Vague I, Mege JL, Mwenechanya J, Tembo M, Grau GE, Molyneux ME: Circulating endothelial microparticles in malawian children with severe falciparum malaria complicated with coma. JAMA 2004, 291:2542-2524.

10. Faille D, El-Assaad F, Alessi MC, Fusai T, Combes V, Grau GE: Platelet-endothelial cell interactions in cerebral malaria: the end of a cordial understanding. Thromb Haemost 2009, 102:1093-1102.

11. Nantakomol D, Dondorp AM, Krudsood S, Udomsangpetch R, Pattanapanyasat K, Combes V, Grau GE, White NJ, Viriyavejakul P, Day NP, Chotivanich K: Circulating red cell-derived microparticles in human malaria. J Infect Dis 2011, 203:700-706.

12. Jy W, Jimenez JJ, Mauro LM, Horstman LL, Cheng P, Ahn ER, Bidot CJ, Ahn YS: Endothelial microparticles induce formation of platelet aggregates via a von Willebrand factor/ristocetin dependent pathway, rendering them resistant to dissociation. J Thromb Haemost 2005, 3:1301-1308.

13. Jimenez JJ, Jy W, Mauro LM, Lens KM, Daghistani N, Dodson HJ, LL H, Ahn Y-S: Activation of endothelial cells (EC) leads to release of inhibitory endothelial microparticles (EMP) and decreased intracellular ADAMTS13 activity. Blood 2006, 108:1810.

14. Jy WLA, Bidot L, Bang J, Ahn E, Horstman LL, Jimenez JJ, Bidot CJ: A significant fraction of ADAMTS13 activity is associated with activated platelets and their microparticles (PMP): implication for regulating ADAMTS13 activity. Blood 2006, 108:1066.

15. Rock $G$, Yousef $H$, Neurath $D, L u$ M: ADAMTS-13 levels in fresh, stored, and solvent detergent treated plasma. Transfus Apher Sci 2006, 35:235-238.

16. Gerritsen HE, Robles R, Lammle B, Furlan M: Partial amino acid sequence of purified von Willebrand factor-cleaving protease. Blood 2001, 98:1654-1661.

17. Gerritsen HE, Turecek PL, Schwarz HP, Lammle B, Furlan M: Assay of von Willebrand factor (vWF)-cleaving protease based on decreased collagen binding affinity of degraded VWF: a tool for the diagnosis of thrombotic thrombocytopenic purpura (TTP). Thromb Haemost 1999, 82:1386-1389.

18. Kawasaki A, Kyogoku C, Ohashi J, Miyashita R, Hikami K, Kusaoi M, Tokunaga K, Takasaki Y, Hashimoto H, Behrens TW, Tsuchiya N: Association of IRF5 polymorphisms with systemic lupus erythematosus in a Japanese population: support for a crucial role of intron 1 polymorphisms. Arthritis Rheum 2008, 58:826-834.

19. Sherry ST, Ward MH, Kholodov M, Baker J, Phan L, Smigielski EM, Sirotkin K dbSNP: the NCBI database of genetic variation. Nucleic Acids Res 2001, 29:308-311.

20. Hanson E, Jood K, Nilsson S, Blomstrand C, Jern C: Association between genetic variation at the ADAMTS13 locus and ischemic stroke. J Thromb Haemost 2009, 7:2147-2148.

21. Burnier L, Fontana P, Kwak BR, Angelillo-Scherrer A: Cell-derived microparticles in haemostasis and vascular medicine. Thromb Haemost 2009, 101:439-451.

22. Faille D, Combes V, Mitchell AJ, Fontaine A, Juhan-Vague I, Alessi MC, Chimini G, Fusai T, Grau GE: Platelet microparticles: a new player in malaria parasite cytoadherence to human brain endothelium. FASEB J 2009, 23:3449-3458.

23. Furlan M, Robles R, Morselli B, Sandoz P, Lammle B: Recovery and half-life of von Willebrand factor-cleaving protease after plasma therapy in patients with thrombotic thrombocytopenic purpura. Thromb Haemost 1999, 81:8-13.

24. Udomsangpetch R, Pipitaporn B, Silamut K, Pinches R, Kyes S, Looareesuwan $S$, Newbold C, White NJ: Febrile temperatures induce cytoadherence of ring-stage Plasmodium falciparum-infected erythrocytes. Proc Natl Acad Sci U S A 2002, 99:11825-11829.

doi:10.1186/1475-2875-13-3

Cite this article as: Kraisin et al:: Reduced ADAMTS13 activity is associated with an ADAMTS13 SNP, fever and microparticles in a malaria-like model. Malaria Journal 2014 13:3. 